Acta Botanica Mexicana 69: 141-147 (2004)

\title{
URTICA PRAETERMISSA (URTICACEAE): UNA NUEVA ESPECIE DEL CENTRO DE MÉXICO
}

\author{
Victor W. SteinmanN \\ Instituto de Ecología, A.C., Centro Regional del Bajío, Apdo. postal 386, \\ 61600 Pátzcuaro, Michoacán, México
}

\begin{abstract}
RESUMEN
Se describe e ilustra Urtica praetermissa (Urticaceae), una nueva especie del centro de México. Se distribuye en el Eje Volcánico Transversal y la Sierra Madre del Sur, en el Distrito Federal y los estados de Guerrero, México y Michoacán. Crece en bosque de Abies, bosque mixto de Quercus-Pinus y matorrales derivados de estos tipos de vegetación en altitudes de 2000 a $3150 \mathrm{~m}$. Contiene dos subespecies: ssp. michoacana y ssp. praetermissa, las cuales se separan por características de las estípulas, connadas en la primera y libres en la segunda. La especie es morfológicamente similar a Urtica mexicana, de la cual se distingue por tener inflorescencias unisexuales o bisexuales con las flores estaminadas y pistiladas mixtas a lo largo de la inflorescencia y aquenios más grandes.

Palabras clave: Eje Volcánico Transversal, México, Sierra Madre del Sur, Urtica, Urticaceae.
\end{abstract}

\begin{abstract}
Urtica praetermissa (Urticaceae), a new species from central Mexico, is described and illustrated. It occurs in the trans-Mexican volcanic belt and the Sierra Madre del Sur in the Distrito Federal and the states of Guerrero, Mexico, and Michoacan. It grows at elevations from 2000 to $3100 \mathrm{~m}$ in forests of Abies and Quercus-Pinus, as well as scrub derived from these types of vegetation. Two subspecies are proposed: ssp. michoacana and spp. praetermissa. These taxa are separated by characteristics of the stipules: connate in the former and free in the latter. The species appears most similar to U. mexicana but differs in possessing larger achenes and inflorescences that are either completely unisexual or bisexual with the staminate and pistillate flowers intermixed along its entire length.
\end{abstract} Urticaceae.

Key words: Mexico, Sierra Madre del Sur, trans-Mexican volcanic belt, Urtica, 
El género Urtica (Urticaceae) contiene alrededor de 80 especies herbáceas distribuidas a través del mundo pero concentradas en ambientes templados del hemisferio septentrional (Friis, 1993). En México se encuentran alrededor de 10 que crecen por lo general en elevaciones altas. Desafortunadamente, la taxonomía de las especies mexicanas aún no está completamente entendida y no existe suficiente literatura para estas plantas, aparte de algunos estudios regionales (por ejemplo, Calderón de Rzedowski, 2001).

Durante la revisión del género para la Flora del Bajío y de Regiones Adyacentes se detectaron especímenes identificados como Urtica mexicana Liebm. o U. chamaedryoides Pursh pero con varias características que permiten reconocer estas plantas como un taxon distinto. Debido a que el material no corresponde con ninguna de las especies anteriormente descritas, se propone como una entidad nueva.

Urtica praetermissa V.W. Steinm., sp. nov.

Herba perennis usque ad $2 \mathrm{~m}$ alta, monoica, stimulosa; stipulae variabiles: connatae, deltoideae vel ovatae, 4.5-8 $\mathrm{mm}$ longae, 2.5-5 mm latae (ssp. michoacana) vel liberae, subulatae, 3-5.5 mm longae, 0.5 a $1.2 \mathrm{~mm}$ latae (ssp. praetermissa); folia opposita, petioli $2.5-6 \mathrm{~cm}$ longi, laminae ovatae, $5-15 \mathrm{~cm}$ longae, 2.5-8.5 latae, basi rotundatae vel cordatae, apice acuminatae, e basi 3-nerviae, margo serratus vel dentatus; inflorescentiae spiciformes elongatae $3-18 \mathrm{~cm}$ longae, unisexuales vel bisexuales; flores staminati: tepala 4, lobi ovati vel oblongi, 0.8-1.4 mm longi, stamina 4, fila 1.8-2.3 mm longa, antherae 0.7-0.9 $\mathrm{mm}$ longae; flores pistillati: tepala 4, inaequales, par parvum anguste oblongum vel lineare, 0.5-0.7 mm longum, $0.2 \mathrm{~mm}$ latum, par grande ovatum, $1.5-2.1 \mathrm{~mm}$ longum, 1.2-1.6 mm latum, stigma penicillatocapitatum, 0.3-0.5 mm longum; achenium ovatum, (1.5)1.6-2.0 mm longum, 1.3-1.6 $\mathrm{mm}$ latum, complanatum.

Hierba perenne hasta de $2 \mathrm{~m}$ de alto, monoica y tal vez también dioica, fuertemente urticante; ramillas densamente puberulentas a pilosas con pelos cortos y suaves y frecuentemente con algunos pelos largos, rectos y rígidos entremezclados; estípulas variables: connadas (por lo menos en la base), deltoides a ovadas, 4.5-8 $\mathrm{mm}$ de largo, 2.5-5 $\mathrm{mm}$ de ancho (ssp. michoacana), o libres, subuladas, 3-5.5 $\mathrm{mm}$ de largo, 0.5-1.2 mm de ancho (ssp. praetermissa), puberulentas a estrigulosas o a veces glabrescentes; hojas opuestas, peciolo de $2.5-6 \mathrm{~cm}$ de largo, con pubescencia como en las ramillas, lámina ovada, de $5-15 \mathrm{~cm}$ de largo, de $2.5-8.5 \mathrm{~cm}$ de ancho, acuminada en el ápice, redondeada a cordada en la base, triplinervada desde la base, margen fuertemente aserrado a dentado, ambas superficies estrigosas a híspidas pero la pubescencia más densa en el envés, cistolitos punctiformes a baciliformes; inflorescencias unisexuales o bisexuales, de 3-18 cm de largo, en forma de una 


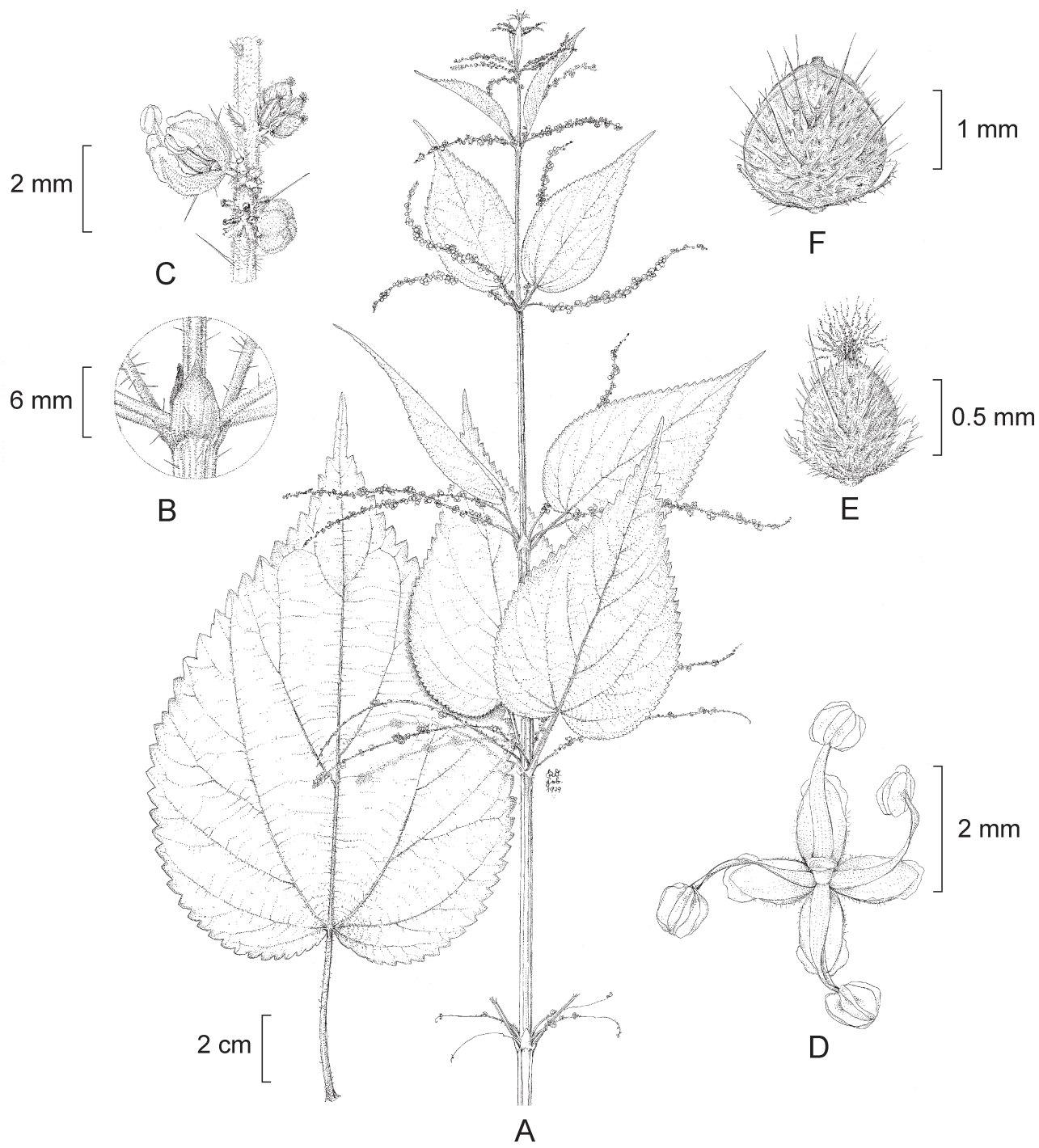

Fig. 1. Urtica praetermissa ssp. michoacana. A. Rama con inflorescencias; B. Estípula; C. Porción de la inflorescencia enseñando flores estaminadas entremezcladas con flores pistiladas; D. Flor estaminada; E. Flor pistilada; F. Aquenio cubierto por los sépalos acrescentes. Ilustrado por Karin Douthit para la Flora Novo-Galiciana; derechos reservados por el Herbario de la Universidad de Michigan y reproducido con su autorización. 
pseudoespiga alargada e interrumpida con las flores agrupadas en pequeños glomérulos, no conspicuamente ramificadas o rara vez con una ramificación lateral corta, inflorescencias bisexuales con las flores estaminadas y pistiladas mixtas a lo largo de la inflorescencia, raquis piloso y frecuentemente con algunos pelos largos, rectos y rígidos entremezclados; flores estaminadas sésiles o en pedicelos hasta de $0.7 \mathrm{~mm}$ de largo, con 4 tépalos y 4 estambres, tépalos unidos en la mitad inferior o libres hasta casi la base, lóbulos oblongos a ovados, de 0.8-1.4 mm de largo, obtusos en el ápice, por fuera estrigulosos y a menudo con algunos pelos largos, rectos y rígidos entremezcaldos, glabros por dentro, filamentos de 1.8-2.3 $\mathrm{mm}$ de largo, anteras oblongas, de 0.7-0.9 mm de largo, pistilodio presente e inconspicuo; flores pistiladas sésiles o en pedicelos inconspicuos hasta de $0.2 \mathrm{~mm}$ de largo, tépalos 4 , libres hasta la base, fuertemente desiguales, los del par pequeño angostamente oblongos o lineares, de 0.5-0.7 $\mathrm{mm}$ de largo, $0.2 \mathrm{~mm}$ de ancho, los del par grande ovados, de 1.5-2.1 mm de largo, de 1.2-1.6 mm de ancho, obtusos en el ápice, por fuera estrigulosos e híspidos, por dentro glabros, estigma penicilado-capitado, de 0.3-0.5 mm de largo; aquenio cubierto por los sépalos acrescentes, anchamente ovoide, ligeramente asimétrico, de (1.5)1.6 a $2.0 \mathrm{~mm}$ de largo, de 1.3 a $1.6 \mathrm{~mm}$ de ancho, fuertemente aplanado, ápice terminando en un pico, base redondeada a truncada, más o menos lisa, de color café claro.

Las flores de los componentes de Urtica son relativamente parecidas entre sí y la delimitación de las especies mexicanas de este género se basa principalmente en características de la inflorescencia, en particular su forma, sexualidad y la disposición relativa de las flores estaminadas y pistiladas. Urtica praetermissa es morfológicamente similar a Urtica mexicana y en ambas especies las inflorescencias consisten de pseudoespigas alargadas. Se diferencian en la disposición relativa de las flores. En U. praetermissa las inflorescencias son unisexuales o bisexuales con las flores estaminadas y pistiladas mixtas a lo largo de la inflorescencia, mientras que en $U$. mexicana las flores estaminadas se presentan en la parte inferior de la inflorescencia abajo de las flores pistiladas. Además, U. praetermissa se puede distinguir de esta última por los aquenios más grandes (1.6-2.0 $\mathrm{mm}$ de largo vs. 1.0-1.3 mm de largo) y una pubescencia de las ramillas más densa. Algunos ejemplares se identificaron como $U$. chamaedryoides, de la cual $U$. praetermissa se diferencia por tener inflorescencias alargadas $(3-18 \mathrm{~cm}$ de largo vs. $0.5-0.9 \mathrm{~cm}$ de largo) y aquenios más grandes (1.6-2.0 mm de largo vs. 1.1-1.2 mm de largo).

Aunque en la mayoría de los ejemplares examinados las inflorescencias son claramente hermafroditas, cabe mencionar que existen algunos con inflorescencias de un solo sexo. Por ejemplo, en Tenorio et al. 1584 las inflorescencias tienen todas las flores estaminadas, mientras que en Cornejo e Ibarra 98 sólo tienen flores 
pistiladas. Faltan observaciones en el campo para determinar si algunos individuos de la especie son unisexuales.

Urtica praetermissa es endémica del centro de México. Se proponen dos subespecies: ssp. michoacana y ssp. praetermissa, las cuales se separan por las características de las estípulas. Además, tienen diferentes áreas de distribución, con ssp. michoacana endémica de Michoacán y ssp. praetermissa prosperando en el Distrito Federal y los estados de Guerrero y México. Se pueden distinguir por las características proporcionadas en la siguiente clave:

1. Estípulas connadas (por lo menos en la base), deltoides a ovadas, de 4.5-8 mm de largo, de 2.5-5 $\mathrm{mm}$ de ancho ssp. michoacana 1. Estípulas libres, subuladas, de 3-5.5 mm de largo, de 0.5-1.2 $\mathrm{mm}$ de ancho ssp. praetermissa

Urtica praetermissa ssp. praetermissa. Tipo: México, Guerrero, along highway between Milpillas (on Highway 95) and Atoyac de Álvarez, $3.7 \mathrm{mi} \mathrm{W}$ of turn-off onto rd to Chichihualco, $2325 \mathrm{~m}$, mature forest, 14 Jan 1979, T. B. Croat 45624 (holotipo MEXU).

Urtica praetermissa ssp. praetermissa se conoce del Distrito Federal y los estados de Guerrero y México, con registros del Valle de México, de la cuenca del Río Balsas y de la Sierra Madre del Sur. Habita en bosque de Abies, bosque mixto de Pinus-Quercus y matorrales derivados de estos tipos de vegetación en altidudes de 2000 a $2750 \mathrm{~m}$. Se ha colectado con flores y frutos en mayo, julio, septiembre y enero. Como es el caso de muchas plantas mexicanas urticantes, su nombre común es "mala mujer" (Ventura 2943).

Paratipos. México. Distrito Federal: delegación de Contreras, Rancho Pachita, Ventura 2687 (MEXU), ibid., Ventura 2943 (MEXU); delegación de Contreras, Los Dinamos, Ventura 3538 (ENCB, MEXU). Estado de México: Cerro de Mamatla, Zacualpan, Matuda et al. 30576 (MEXU).

Urtica praetermissa ssp. michoacana V.W. Steinm., ssp. nov. Tipo: México, Michoacán, municipio de Angangueo, vertiente O de Barranca Honda, Ejido Cerro Prieto, 3100 m, bosque de Abies en cañada, 15 Mzo 1987, H. Díaz B. 3593 (holotipo IEB, isotipo ENCB).

Differt a ssp. praetermissa stipulis connatis. 
Como anteriormente se mencionó, Urtica praetermissa ssp. michoacana se distingue por la forma de sus estípulas, las cuales son connadas. Este atributo es particularmente notable ya que es el único taxon mexicano del género que presenta este rasgo, aunque el mismo aparece en varias especies asiáticas (Jiarui et al., 2003).

La subespecie se conoce hasta ahora sólo de la parte norte de Michoacán, aunque es posible que exista también en las regiones adyacentes del estado de México. Esta área forma parte del Eje Volcánico Transversal, y la planta se encuentra tanto en la vertiente de la cuenca del Río Balsas como de la del Río LermaSantiago. Habita en bosque de Abies y bosque mixto de Pinus-Quercus en altitudes de 2300 a $3150 \mathrm{~m}$. Florece por los menos desde septiembre hasta abril. Como en el caso de la mayoría de las especies mexicanas de Urtica, el nombre común es "ortiga" (Cornejo e Ibarra 98).

Paratipos. México. Michoacán: municipio de Ocampo, Santuario de la Mariposa Monarca El Rosario, 19³5'28" N, 100¹5'54" O, Cornejo e Ibarra 98 (IEB); municipio de Uruapan, pedregal al E de Capacuaro, García y Pérez 3191 (IEB); municipio de Zinapécuaro, camino viejo hacia la presa La Gachupina, Jasso 303 (ENCB, IEB); municipio de Ocampo, El Rosario, 23 II 1988, Mejia s. n. (IEB); municipio de Tlalpujahua, cerca de Pichardo, $5 \mathrm{~km}$ al SW de San Francisco Los Reyes, Rzedowski 46219 (ENCB, IEB); municipio de Senguio, El Salto, cerca de La Capilla del Ocotal, Rzedowski 48497 (IEB); municipio de Zitácuaro, $7 \mathrm{~km}$ al E de Zitácuaro, carr. Zitácuaro-Toluca, Macho de Agua, Tenorio et al. 1584 (IEB, MEXU).

\section{AGRADECIMIENTOS}

Agradezco a Graciela Calderón de Rzedowski, Paul Fryxell y Sergio Zamudio por la revisión crítica del escrito; a los responsables de los herbarios ENCB, IEB y MEXU por proporcionar especímenes; a los Drs. William R. Anderson y Rogers McVaugh (MICH) por permitir el uso del dibujo no publicado de su Flora NovoGaliciana; a la Comisión Nacional para el Conocimiento y Uso de la Biodiversidad, al Consejo Nacional de Ciencia y Tecnología, así como al Instituto de Ecología, A. C. (cuenta 902-07) por el apoyo económico recibido. 


\section{LITERATURA CITADA}

Jiarui, C., L. Qi, I. Friis, C. M. Wilmot-Dear y A. K. Monro. 2003. Urticaceae. In: Flora of China Editorial Committee (eds.). Flora of China Vol. 5. Ulmaceae through Basellaceae. Missouri Botanical Garden Press. St. Louis. pp. 76-189.

Calderón de Rzedowski, G. 2001. Urticaceae. In: Rzedowski, G. C. de, J. Rzedowski y colaboradores. Flora fanerogámica del Valle de México. 2a. ed. Instituto de Ecología, A.C. y Comisión Nacional para el Conocimiento y Uso de la Biodiversidad. Pátzcuaro. pp. 93-99.

Friis, I. 1993. Urticaceae. In: Kubitzki, K., J. G. Rohwer y V. Bittrich (eds.). The families and genera of vascular plants II. Flowering plants. Dicotyledons. Magnoliid, Hamamelid and Caryophyllid families. Springer-Verlag. Berlin y Heidelberg. pp. 612-630.

Recibido en agosto de 2004. Aceptado en noviembre de 2004. 